\title{
Measurment methods for understanding water uptake processes in polymers
}

\author{
Paul Gierth, Uta Gierth, Lars Rebenklau, Michael Schneider \\ Fraunhofer IKTS, Winterbergstrasse 28, 01277 Dresden, Germany \\ paul.gierth@ikts.fraunhofer.de
}

\begin{abstract}
Summary:
Harsh environmental conditions may cause corrosion processes in electronics. They are mainly induced by water uptake of polymer embedding, housing or molding compounds. Therefore, longtime stability of these materials is critical for many applications. This work is focusing on demonstration and discussion of suitable measurement methods to determine the water uptake of polymers. Electrochemical impedance spectroscopy (EIS) and Fourier Transform Infrared spectroscopy (FTIR) were used to investigate polymer degradation before and after multiple loading scenarios. Degradation models could have been derived from these results.
\end{abstract}

Keywords: Electrochemical impedance spectroscopy, Fourier transform infrared spectroscopy, polymers, water uptake, degradation

\begin{abstract}
Motivation
Main purpose of polymers are corrosion protection of conductors and electronic components. Therefore, the longtime stability of these materials against different loading scenarios is critical for many applications. Usually, modern test scenarios just focus on pass or fail results, e.g. mechanical characterization [1]. Such tests are regularly performed before and after defined loading scenarios like damp heat, temperature cycling or pressure cooker treatment. Unfortunately, the results only summarize the accumulated alteration at the time of test. A deep understanding of the fundamental reaction processes or kinetics can't be achieved in that way but is necessary for further improvement of materials and technologies. In addition to conventional shear test, the Methods EIS and FTIR were applied.
\end{abstract}

\section{EIS and FTIR for electronic industry}

EIS and FTIR is commonly used in corrosion research. By means of EIS the determination of electrical impedance as a function of the measuring frequency can be realized. Standardly, this method is applied to investigate material characteristics, such as dielectric permittivity and capacitance. In insulating materials, a capacitive characteristic appears, whereas in electrical conductors an ohmic characteristic dominates. Polymers present a shifting characteristic between these two main states in dependency of their degradation state. A fast condition check is possible, especially water uptake, due to voids, blister, or insufficient cross-linking of the polymer structure. In addition, FTIR helps to investigate the molecular changes by stimulation of functional groups. For example, formation of intramolecular hydrogen bonds within the polymer network is detectable. Additionally, analyzing destruction of chemical bonds is possible.

\section{Experimental}

Two kinds of epoxy coatings were selected and cured on printed circuit boards (PCB) with electroless nickel immersion gold (ENIG) finishes. These samples were aged for up to $96 \mathrm{~h}$ in a pressure cooker test or up to 14 days in a damp-heat-treatment and further inspected regarding their shear strength stability. The degradation process was investigated with EIS and corelated with FTIR measurement, but discussion is focused on EIS.

\section{Results}

Shear strengths are summarized in Fig. 1 and 2.

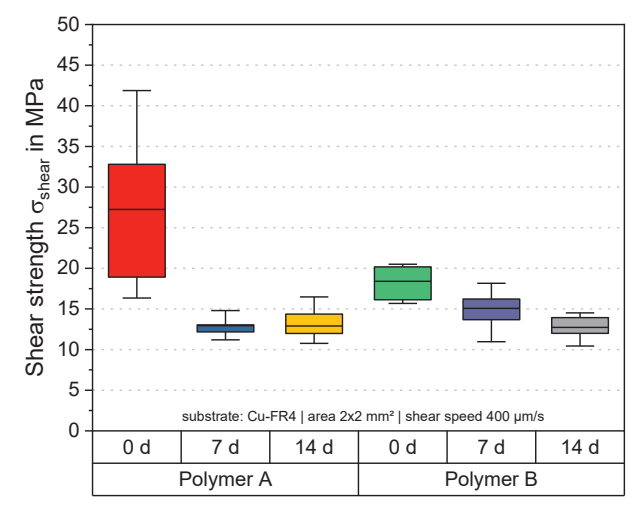

Fig. 1. Shear strengths of epoxies on ENIG surfaces before and after damp heat treatment 


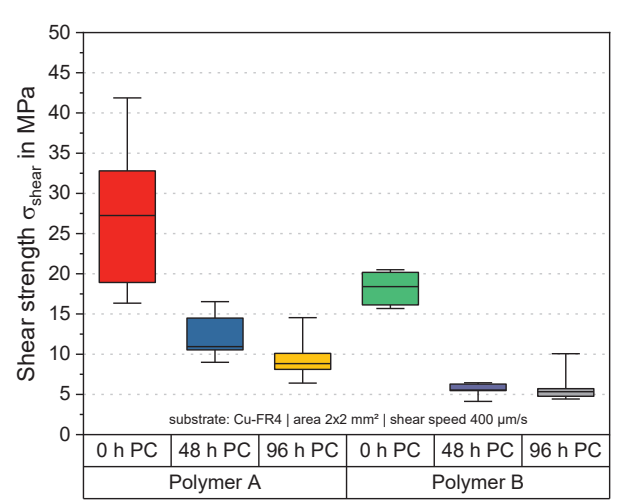

Fig. 2. Shear strengths of epoxies on ENIG surfaces before and after pressure cooker treatment (PC)

Both loading scenarios show strong mechanical degradation of the inspected epoxies. The pressure cooker treatment leads to a faster degradation overall. The optical inspection of all fracture zones shows adhesive as well as cohesive failure mechanisms. Consequently, no clear degradation mechanism could be observed. Optical inspection of the polymers revealed that polymer $A$ occurs defects like blisters, pinholes, or inclusions in certain regions. Polymer $B$ seems to be nearly free of defects.

Samples were analyzed with EIS before and after PCT to obtain a better understanding of the degradation mechanism.

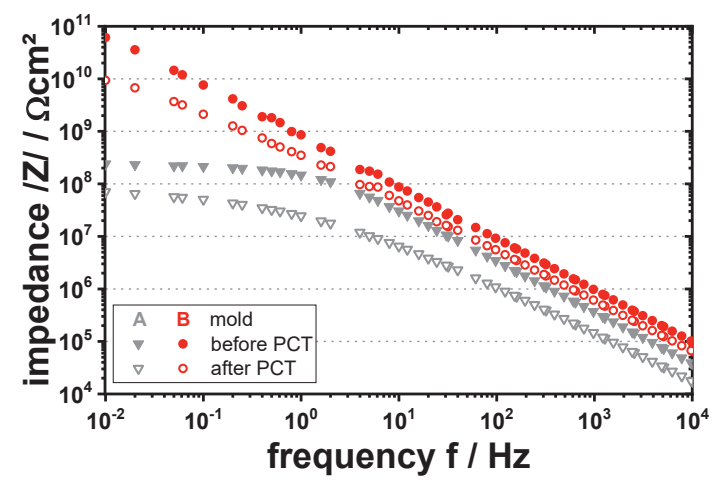

Fig. 3. EIS: Impedance of polymer $A$ and $B$ before and after pressure cooker treatment (PC).

The change of capacitive reactance on high frequency region during different loading scenarios is used to calculate water uptake WU of the material by using eg. (1) according [2].

$W U=\frac{\lg \left(\frac{C_{t}}{C_{o}}\right)}{\lg 80} \cdot 100($ Vol. $\%)$

The water uptake was calculated to approximately $21 \mathrm{Vol} . \%$ for polymer A and $11 \mathrm{Vol} . \%$ for polymer $\mathrm{B}$. The different results indicate different water penetration scenarios. For the nearly perfectly cured Polymer B, liquids could only penetrate the material by a homogeneous diffusion across the material. A polymer with several surface defects $(A)$ offers the possibility for inhomogeneous penetration of any kind of liquids into deeper material regions along preferred paths and accelerates the degradation in this way. Selective penetration could be found in phase shift of the impedance during EIS measurement and was used to generate following equivalent circuits according $[4,5]$.

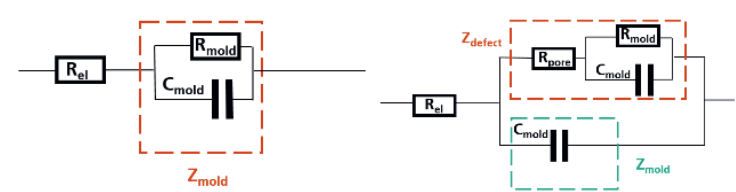

Fig. 3. Equivalent circuits of polymers on metallic surfaces without surface defects (left) and with liquid penetrated pores (right).

Therefore, pre-existent defects previously detected by optical microscopy of polymer A could clearly been seen in the initial EIS measurement (lower frequencies) and their influence on environment stability could be described using the shown methods. In that way, a better understanding of different degradation mechanisms could be found without destruction of the samples, which demonstrates the usability of this measurement method as material and application controlling method.

For a complete understanding of the ongoing degradation mechanisms during the applied loading scenarios FTIR inspections must be applied. The obviously change in intensity ratio of characteristic bands provides information like an increase of hydroxide ion content or decreasing of epoxide bond. That indicates a significant change of the material during the loading scenario. These results are appropriate to generate better understanding in material degradation mechanism and material state monitoring over product lifetime cycles. Therefore, methods like EIS and FTIR are highly recommended to be transferred from the corrosion research to the electronic industries.

\section{References}

[1] Military Specification (MIL)-883, Method 2019.9 Die shear strength, revision E, Department of Defense, 1991.

[2] D. M. Brasher, A. H. Kingsbury, electrical measurment in the study of immersed paint coatings in metal, Journal of Applied Chemistry, 1954.

[3] U. Rammelt, habilitation thesis, TU Dresden 1991.

[4] G. Grundmeier et al.: Electrochim. Acta 45 (2000) 2515 